\title{
Innovation numérique et transformation structurelle des économies africaines francophones, opportunités risquées pour le développement?
}

\section{Digital innovation and structural transformation of French-speaking African economies: risky opportunities for development?}

\author{
Kako Nubukpo ${ }^{1}$, Ludovic Temple ${ }^{23}$, Chloé Alexandre ${ }^{23}$ \\ ${ }^{1}$ Université de Lomé, Togo, kakonubukpo@yahoo.com \\ ${ }^{2}$ Cirad Umr Innovation, Montpellier France, ludovic.temple@cirad.fr et chloe.alexandre@cirad.fr \\ ${ }^{3}$ Université de Montpellier F-34090 Montpellier France
}

RÉSUMÉ. Du fait de la rapidité et l'ampleur des changements qu'il induit en Afrique subsaharienne, le numérique transforme les modèles politiques, économiques, sociaux et culturels traditionnels. Mais à qui ces changements profitent-ils ? Ces technologies participent-elles à une transformation structurelle profonde des économies et sociétés ouest-africaine, en rupture avec les modèles de développement observés jusqu'alors ? Cet article synthétise l'état des connaissances mobilisables sur la nature des relations entre le numérique et la transformation structurelle des économies africaines (mise en commun des ressources, industrialisation des services, risques d'une gouvernance globalisée) et sur l'émergence à plusieurs niveaux de cadres de régulation de ce secteur technologique. Nous mobilisons pour ce faire différentes expériences empiriques dans la gouvernance de l'espace francophone, dans l'accompagnement des politiques de recherche et d'innovation nationales et dans l'analyse des conditions du développement du numérique dans le secteur agricole. Nous documentons ainsi le champ des opportunités et des risques que constitue l'exploitation de ces technologies dans le cadre du développement de l'Afrique francophone.

ABSTRACT. Digital technologies are inducing rapid and profound changes in Sub-Saharan Africa and are transforming traditional political, economic, social and cultural models. But who is benefiting from these changes? Are these technologies contributing to a structural transformation of West African economies and societies, breaking with the development models which have prevailed so far? This article summarizes the state of our knowledge on the nature of the relationships between digital technologies and the structural transformation of African economies (pooling of resources, services industrialization and risks of a globalized governance) and on the emergence of multilevel regulation frameworks in this technological sector. To do so, we build on various empirical experiences, respectively in the French-speaking area's governance, in the support of national research and innovation policies, and in the analysis of digital development in the agricultural sector. We thus document the scope of opportunities and risks of digital expansion with regards to the development of French-Speaking African countries.

MOTS-CLÉS. Afrique - Numérique - Innovation.

KEYWORDS. Africa - ICT - Innovation.

\section{Introduction}

La rapidité et l'ampleur des changements induits par le numérique en Afrique subsaharienne situent la numérisation comme un moteur de l'innovation pour l'économie et la société. Sur le plan économique, les Technologies de l'Information et de la Communication (TIC) sont source d'accroissement de la productivité du travail, du capital, de la valeur ajoutée qui stimulent la demande de biens et de services. Elles constituent un levier potentiel pour diminuer les inégalités d'accès à certains services dans l'administration publique, la santé (télé-diagnostic), l'éducation (bibliothèques virtuelles, e-learning), les services financiers [RIC 17]. Elles automatisent et dématérialisent progressivement la nature du travail. Elles induisent des phénomènes de « destruction créatrice » qui renouvellent la création d'emplois dans différents secteurs et les diversifications sectorielles et intra-sectorielles. De nouveaux services sont créés pour les usagers, des services anciens sont rendus plus efficaces par ces technologies. Le numérique décline en soit 
les promesses d'une nouvelle grammaire du progrès qui implique un changement de paradigme dans la conception du changement technologique et de ses relations avec le développement. Ainsi les programmes de coopération mis en œuvre dans l'espace francophone visent à accompagner les acteurs et les institutions pour mettre en place les prérequis nécessaires à la mobilisation du numérique comme levier du développement aux plans micro, méso et macro-économiques. Cet engouement technologique cache cependant les risques qui accompagnent tout changement technologique majeur [GOD 17]. Il incite donc à documenter la mise en place de politiques de régulation de la révolution numérique. Au regard d'une analyse des relations entre le numérique et les transformations structurelles des économies en développement, nous proposons de documenter le champ des opportunités et de risques liés à l'exploitation de ces technologies pour le développement, en contextualisant sur la base d'expériences empiriques dans les pays d'Afrique de l'Ouest francophones.

La notion de transformations structurelles peut renvoyer dans les travaux sur les transitions sociotechniques à la caractérisation des éléments qui structurent l'environnement macro-économique [GEE 02]. Dans une posture d'institutionnalisme méthodologique, cet environnement [CAD 16] résulte principalement de l'hybridation entre deux modes de gouvernance des politiques de développement [HUG 80]. La première est historiquement donnée par les pays de l'OCDE (Organisation de Coopération et Développement Economiques) : anciennes politiques coloniales d'asservissement du $19^{\text {ème }}$ siècle, néo-colonialisme industriel d'après-guerre [AMI 73], programmes d'ajustements structurels des années 80 [UZU 11], renouvellement des objectifs de développement mondiaux [EGG 18]. La seconde émerge de la structuration des politiques nationales dans une diversité de situations au regard de la construction et de l'affirmation des Etats [RAZ 17]. Trois angles d'analyse sont proposés pour ces transformations.

Le premier est celui de l'industrialisation, censée générer des mécanismes où les produits d'un secteur ou d'une branche deviennent les intrants d'un(e) autre nourrissant des intégrations intersectorielles et donc des dynamiques endogènes de transformations technologiques liées à ces intégrations. Il est pour partie tributaire de variables macro-économiques : différentiel du coût du travail, investissements publics, capacités d'apprentissage nationales [CAS 15]. Cette industrialisation bénéficie de la massification des marchés intérieurs liée aux transitions sociodémographiques [LOS 12], la croissance des villes et des demandes alimentaires [DUR 17, FOU $18]$.

Le deuxième est celui de la gouvernance macro-politique qui lie la croissance économique aux mécanismes de réduction ou d'augmentation des inégalités sociales [STI 14] et d'émergence de classes moyennes. Cette gouvernance nourrit l'accroissement des diasporas et des stratégies entrepreneuriales d'investissement des capitaux de rente dans le système productif national [CAS 17], au lieu de favoriser la spéculation dans les opportunités financières mondialisées offertes par les pays industriels ou émergents [BAY 06].

Le dernier est celui de la spécialisation du continent africain dans l'approvisionnement en matières premières : minerais, énergie, produits agricoles [DAV 02, NUB 11, NUB 12] qui conduit à une dépendance de la croissance économique aux instabilités des marchés internationaux. Elle favorise aussi la constitution de rentes génératrices d'accroissement d'inégalités sociales et de gouvernances publiques défaillantes. Cette spécialisation se fait donc au détriment des mécanismes intégratifs économiques et sociétaux qui permettraient un développement répondant aux aspirations sociétales des populations locales. Ces aspirations ne sont en effet pas forcément alignées avec les idéaux consuméristes incarnés par les vitrines des pays industriels.

Ces transformations structurelles suivent plusieurs trajectoires d'évolution. 
L'une prolonge une industrialisation fondée sur les modèles technologiques des pays industriels selon différentes options [DEB 71]. Elle repose sur transferts technologiques industriels. Dans un contexte de faible régulation ou fonctionnalité par les politiques publiques nationales, elle porte les risques d'une destruction ou " consommation » de ressources naturelles (eau, biodiversité, énergie, biomasse, etc.).

L'autre propose une diversification structurée par les opportunités qui s'ouvrent dans les intégrations économiques régionales, mais aussi dans l'économie de services. L'Afrique ne pourraitelle pas entrer dans la modernité du développement en sautant l'étape industrielle, réputée destructrice des cadres de vie environnementaux, sanitaires et sociaux ? Une telle perspective est sans doute utopique, mais la rupture du paradigme de développement ne serait-elle pas salutaire ? En l'occurrence, les situations d'urgences que pose la conjonction des enjeux environnementaux, démographiques et alimentaires [FAU 18] interrogent la promesse que propose la « révolution» numérique.

Au regard de cette mise en compréhension probablement partielle des déterminants structurels du développement de l'Afrique sub-saharienne, nous analysons les contributions que portent les technologies numériques au prisme de deux questions complémentaires :

- la nature des relations entre le numérique et la transformation structurelle des économies africaines. Nous situerons ainsi en quoi le numérique, tout en étant objet lui-même d'innovation, est un déterminant ambivalent de ces transformations dans le renouvellement des modes de gouvernance de l'innovation qu'il génère : globalisation des systèmes de connaissances mobilisables et nouvelles dépendances à l'égard de la concentration des firmes dans ce secteur.

- la diversité des échelles dans les mécanismes d'évaluation et de régulation du numérique (normes d'usage, règlementations) au niveau : des politiques publiques nationales, instances intergouvernementales ou de la société civile.

- Nous mobilisons les bases de connaissances issues de trois situations empiriques. La première résulte de l'expérience acquise dans des fonctions de gouvernance de politiques publiques nationales et régionales au sein de l'Organisation Internationale de la Francophonie. La deuxième, de l'implication dans l'analyse des conditions d'émergence des politiques nationales de recherche et d'innovation [TEM 17]. Et la troisième, de l'analyse, des relations entre le numérique et les activités d'accompagnement de l'innovation dans le secteur agricole [ALE 19].

\section{Les relations entre le numérique et la transformation structurelle des économies africaines}

\subsection{Mise en commun des ressources cognitives}

L'extension du numérique serait un levier central de la globalisation d'accès des populations aux bases de données ouvertes, mais surtout à des ressources éducatives libres. Elle contribuerait à la démocratisation des conditions d'accès à différentes formes de connaissances et d'informations et concourrait ainsi à promouvoir une philosophie collective du partage gratuit de ces ressources. De fait, le numérique, dans cette orientation, serait un moteur favorisant la création de biens communs sur des ressources autrefois privatisées, ou sur lesquelles les conditions d'accès institutionnel ou par les droits pouvaient créer de l'exclusion. Cette contribution potentielle est cependant controversée. Ainsi la transition numérique accroît certes les espaces collaboratifs, notamment via les réseaux sociaux (Facebook, WhatsApp, etc.) ou des plateformes créant de nouveaux réseaux d'acteurs [FUH 17]. Mais ceci se fait aussi de plus en plus au détriment des réseaux de coopération structurés par les processus de construction collective de connaissances [LAU 18]. Le numérique en créant l'illusion d'une connaissance déjà constituée qu'il suffit de mettre en partage, entrave les mécanismes de coopération nécessaires à la création de nouvelles connaissances. Il donne l'illusion que l'échange 
virtuel de ce qui existe déjà peut remplacer les situations d'échanges basées sur la rencontre, la confrontation ou dispute sur ce qui n'existe pas encore. La transition numérique serait alors un facteur limitant majeur de la transition écologique [LAU 18]. Le numérique, et plus particulièrement les TIC offrent de réelles opportunités pour produire, gérer et partager information et connaissances [HIS 13], notamment dans le secteur agricole [NNA 12, VAN 15, ALE 18]. Selon la Banque mondiale (2016), ce secteur emploie environ 65\% de la population active en Afrique. La demande en conseil et formation agricole y est donc forte, chez les jeunes mais aussi les adultes. Les TIC sont donc promues comme des outils pouvant améliorer la couverture du conseil (en offrant des services à distance), sa pertinence (en produisant des informations plus fiables et spécifiques aux attentes des agriculteurs) ou son efficacité (en numérisant des tâches telle la collecte de données sur papier).

Les échecs des initiatives 'ICT4D' (Information and Communication Technologies for Development) sont cependant nombreux [HEE 02].

Par ailleurs, les impacts de ces technologies sur la production, les apprentissages, les revenus et les conditions de vie des agriculteurs sont encore difficilement perceptibles [AKE 16]. Au Burkina Faso, la mobilisation des TIC est présentée comme un des piliers pour améliorer les services d'accompagnement de l'innovation au sein des organisations fournissant du conseil agricole principalement l'Etat, les organisations de producteurs (OP), les organisations non gouvernementales (ONG). Cependant, ces services de conseil agricole numériques demeurent émergents [ALE 19]. Plusieurs barrières se posent à leur développement : infrastructures peu développées (réseau téléphonique et internet ; énergie), ressources financières limitées des opérateurs de conseil, faible niveau d'alphabétisation des agriculteurs et faible capacité à payer des usagers. A ceci s'ajoutent des défis tenant à la création de partenariats et à la gestion de ces relations inter-organisationnelles. Développer un service numérique nécessite en effet des collaborations entre des organisations, locales et internationales, issues de divers secteurs (ingénierie, conseil, aide au développement, téléphonie, ...). Ces collaborations inter-organisationnelles, bien qu'indispensables, ralentissent le développement de ces services. Elles nécessitent de constantes négociations entre ces partenaires, notamment pour trouver un modèle d'affaire et un partage des bénéfices appropriés. Un autre défi majeur pour développer ces services de conseil numériques tient à la production d'un contenu informationnel pertinent. Produire des informations spécifiques aux besoins des agriculteurs nécessite un investissement significatif que toutes les structures ne sont pas capables de fournir [ALE 18]. A l'heure actuelle, les TIC les plus accessibles aux agriculteurs (téléphones simples) servent principalement à partager des informations simples sur le contexte climatique (météo) et économique (prix et marchés) ou à diffuser des techniques de production standardisées. Etant donnés les échecs des approches de «transfert de connaissances » [ROG 88], on peut donc douter de l'efficacité de ces services pour favoriser des réels apprentissages chez des agriculteurs faisant face à des situations complexes. Les outils connectés (smartphones, tablettes, ordinateurs) offrent par contre des usages intéressants. La création de systèmes d'information peut alléger le travail des conseillers (en numérisant certaines tâches telle la collecte de données) et offrir des renseignements cruciaux pour le pilotage des services de conseil. Ces outils offrent également un fort potentiel pour co-construire des connaissances qui répondent aux attentes spécifiques des agriculteurs. C'est le cas notamment des réseaux sociaux et des plateformes numériques, favorisant des échanges entre agriculteurs et facilitant les interactions entre savoirs "d'experts » et savoirs " paysans ». La co-production des outils de conseil et des connaissances avec les agriculteurs reste cependant embryonnaire. Le contenu informationnel partagé par les services de conseil numérique est majoritairement produit par des ONG et des centres de recherche internationaux, qui impliquent rarement les agriculteurs.

L'émergence d'initiatives locales portées par des agriculteurs est plus rarement observée, notamment via l'utilisation des réseaux sociaux [SUC 16; MIL 19]. Cependant, les administrateurs de ces réseaux témoignent des efforts (plus en termes de temps que d'argent, les services étant 
gratuits) pour s'assurer de la fiabilité du contenu partagé selon ce mode collaboratif ('crowdsource').

Ces nouvelles formes de conseil via les réseaux sociaux imposent aussi de sécuriser la production du contenu informationnel afin d'éviter les risques d'instrumentation par des firmes. On peut par exemple observer la promotion de fiches techniques pour l'usage d'herbicides ou insecticides, sans que cela soit forcement justifié par des travaux d'expérimentation agronomiques référencés. En dehors des initiatives d'utilisation des réseaux sociaux portées par de jeunes agriculteurs, les fournisseurs de services relatent leurs difficultés à convaincre les acteurs disposant d'informations d'intérêt public (Etat, recherche et ONG notamment) de les partager en libre accès. Comme le constate un des gestionnaires du projet E-Burkina, géré par l'Agence Nationale de Promotion des TIC (ANPTIC) et financé par la Banque Mondiale, les initiatives 'Open Data' ont des difficultés à percer. Ce constat est partagé par les fournisseurs de contenu cherchant à créer des plateformes alimentées de manière collaborative par les acteurs du monde rural et de la recherche. Une autre limite de ces nouveaux modes de conseil via les réseaux sociaux ou les plateformes numériques relève de l'accès par les agriculteurs aux outils connectés. En effet, on recensait seulement 11\% d'utilisateurs d'internet au Burkina en 2016, tous secteurs confondus. L'utilisation du smartphone, de tablette ou d'ordinateur reste donc réservée à une élite. Or une étude sur 15 services de conseil numérique au Burkina montre que seuls 6 sont accessibles via des téléphones simples, pourtant les media les plus couramment utilisés par les agriculteurs [ALE 19]. Les services de conseil agricole intégrant le numérique constituent donc une innovation de niche et l'utilité de certains services, transférant des connaissances très standardisées et normatives, reste questionnable.

\subsection{L'entrepreneuriat numérique ou une nouvelle industrialisation par les services}

La jeunesse africaine fait preuve d'une forte appétence pour la production de solutions numériques au plan micro-économique. La Francophonie (OIF) a par exemple développé une plateforme de financement participatif, «Finance Ensemble», qui permet de mobiliser des ressources pour financer quelques applications numériques. Ceci montre que les jeunes africains ont à cœur la question sociale car la plupart des solutions primées lors des différents concours renvoie à une volonté de recherche d'utilité sociale.

Plusieurs exemples empiriques mis en œuvre par de jeunes entrepreneurs illustrent ces situations comme par exemple au Burkina Faso : une cartographie des centres de santé a été primée par la Francophonie ; une application nommée «E Civil » a été développée pour enregistrer automatiquement le bébé dès sa naissance au niveau de l'état civil (ce qui permet d'améliorer le suivi des enregistrements des naissances à l’Unicef).

Au Burkina Faso, les démarches de conception agile et les « hackatons » sont fortement promus par le Ministère du Développement de l'Economie Numérique et des Postes pour soutenir les jeunes entrepreneurs innovants. Dans le domaine agricole, un hackaton a permis à 3 lauréats de développer respectivement une plateforme permettant la surveillance, l'alerte précoce et la prise en charge de ravageurs des cultures ( Song koobo ») ; un système automatisé de conservation de produits agricoles («Smart conservation») et une plateforme de commercialisation des produits agropastoraux ( $\ll$ BeogoFarm »).

Comme nous l'avons vu dans le cas du Burkina Faso, les apports du numérique sur la construction de connaissances utiles dans le domaine agricole restent encore embryonnaires. Cependant la mobilisation de ces technologies transforme progressivement les systèmes d'accompagnement des agriculteurs.

De nouveaux acteurs se positionnent au sein des systèmes d'innovation agricoles, principalement des entreprises de développement informatique, des fournisseurs de logiciels, des agrégateurs de données et des opérateurs téléphoniques. Ces entreprises sont en majorité basées dans des pays 
industrialisés, mais on note également la création d'entreprises et d'ONG locales spécialisées dans le développement de solutions numériques pour le secteur agricole. Cependant, ces organisations locales éprouvent des difficultés à faire face à la concurrence des entreprises étrangères du même secteur [ALE 19]. Ce constat de la faible présence des acteurs locaux dans le secteur du numérique (hardware et software) dépasse le seul secteur agricole, mais le développement de collaborations internationales peut permettre aux entreprises locales de renforcer sur le moyen terme les compétences qui leur manquent pour s'imposer dans le secteur. Ainsi dans le projet E-burkina (visant notamment à développer et gérer les systèmes d'information des différents ministères), les offres retenues sont celles où des entreprises locales s'associent à des entreprises européennes, d'Afrique du nord ou des Etats-Unis.

\subsection{Les risques d'une gouvernance globalisée des écosystèmes de l'internet}

Tout comme l'espace physique fait l'objet de contestations diverses, le cyber espace est l'objet de convoitises, dont certaines passent par la détention privée de noms de domaine indispensables pour accéder à la cyber nationalité. Ces extensions de noms de domaine (.tg pour le Togo, .ml au Mali) ont appartenu ou sont toujours détenus par des sociétés privées, ayant déposé un droit de propriété il y a une vingtaine d'années. Au Mali, le propriétaire de l'extension .ml est basé aux Pays-Bas. D'autres pays ont cependant engagé un travail de négociation pour racheter ces noms de domaine. Ainsi au Burkina, l'extension '.bf' est désormais gérée par une institution nationale, l'Autorité de Régulation des Communications et des Postes, en collaboration avec l'opérateur téléphonique Onatel.

On constate par ailleurs que se reproduisent dans le cyberespace, les mêmes enjeux de souveraineté qu'on peut trouver dans l'espace physique. Derrière tout ce qui renvoie à l'innovation numérique sur le plan macro, on retrouve des enjeux liés à l'espace physique. Dans le cas de la cyber-sécurité on observe une corrélation très forte entre la capacité d'un Etat à maîtriser son cyberespace et sa capacité à assurer la sécurité physique de ses ressortissants [GHE 12]. Avant même les attentats physiques, il y a une bataille qui se livre au niveau du cyberespace.

A titre d'exemple, nombre de ministres, parlementaires et hauts cadres des administrations africaines travaillent et correspondent au quotidien en utilisant des adresses mails comme yahoo ou gmail. Ceci peut être hautement préjudiciable à la sécurité au niveau d'un Etat, compte tenu du faible degré de confidentialité des informations échangées sur ces plateformes. Il y a donc un enjeu de renforcement de capacités pour que les Etats africains puissent contrôler la gestion et la gouvernance de leur écosystème numérique.

Avec l'aide apportée par des bailleurs, concédant au pays des prêts à taux concessionnel, le Burkina Faso a significativement investi pour renforcer les infrastructures et services numériques du gouvernement, et pour développer les capacités nécessaires à la gestion de ces cyberespaces. Le numérique y est perçu comme un facteur indispensable à l'édification d'une administration transparente et efficace.

Le Burkina a créé dès 1995 un réseau interne à l'administration, le Resina (RESeau Informatique National de l'Administration), ceci faisant de lui un pays pionnier dans la sous-région. Suite aux améliorations et élargissements successifs, ce réseau permet aux fonctionnaires sur l'ensemble du territoire de bénéficier de services sécurisés (applications métiers de l'administration, navigation internet, messagerie électronique, téléphonie sur IP). D’autres services numériques seront développés pour faciliter le travail de l'administration. La création d'un «G-Cloud » permettra de mutualiser les infrastructures de stockage commun à l'ensemble des organes de l'administration, une première en Afrique de l'Ouest. L'application «E-conseil des Ministres » a quant à elle été développée par une société burkinabé dans le but de numériser les rapports mobilisés et décisions prises hebdomadairement. L'application, censée améliorer l'efficacité de ces rencontres et limiter la 
fuite d'informations, n'est cependant pas utilisée. La complexité de la plateforme créée ne facilite pas son utilisation. De plus, le fort turn-over des Ministres amenés à l'utiliser implique d'investir à chaque vague de renouvellement dans du matériel et des formations. Selon l'ANPTIC, bras opérationnel de l'Etat pour gérer ses systèmes d'informations, les enjeux de souveraineté sur le cyberespace, réclamant par exemple le développement de centres de stockage de données sur le territoire national, ne sont pas encore la priorité pour le pays. L'urgence est bien de développer des infrastructures et services viables pour l'administration, les entreprises et les citoyens et de renforcer les compétences des développeurs et des utilisateurs de ces solutions numériques. Concernant les enjeux de cyber-sécurité, le Burkina est en cours de transformation de son cadre législatif afin de définir la nature des infractions en lien à l'utilisation du numérique et les sanctions qu'elles entraînent. Ce nouveau cadre n'est cependant pas encore opérationnalisé.

\section{L'analyse multi-niveaux : Le passage à l'échelle}

L'observation de l'entrepreneuriat numérique en Afrique met en évidence l'existence de beaucoup d'imagination, de créativité mais des difficultés à passer à l'échelle. Ainsi, la nature des opportunités et risques que crée le numérique dans l'innovation frugale à l'échelle d'un pays ou au niveau d'une entreprise, questionne les conditions institutionnelles de son activation au service du développement. Quels sont finalement les éléments fondamentaux que l'innovation liée au numérique porte au regard de la transformation structurelle des économies africaines ? Quatre peuvent être mis en évidence, correspondant à autant de niveaux d'analyse.

\subsection{Le niveau des politiques publiques nationales}

L'extension du numérique ouvre de nouveaux enjeux dans la globalisation des mécanismes de construction et gouvernance des politiques publiques. La co-construction des politiques, programmes et projets à l'échelle nationale est au centre des mécanismes futurs de la trajectoire de développement en Afrique. Les outils numériques peuvent faciliter la construction plus efficace de politiques publiques. Les bibliothèques numériques et cours en ligne ont ainsi significativement renforcé les compétences des fonctionnaires des administrations ouest-africaines. Cependant, il convient de s'interroger sur l'orientation idéologique des organisations qui créent ces outils de formation. Le Fonds monétaire international (FMI) a par exemple longtemps négocié avec la Francophonie pour la traduction de ses cours de programmation financière (essentiellement disponibles en anglais) et leur diffusion en ligne en français. L'objectif était de construire des MOOC en français pour les cadres des administrations économiques et financières francophones. Les cours y ont été rédigés essentiellement selon l'application de dogmes néo-libéraux dans la régulation des politiques monétaires internationales. Le numérique dans cet exemple devient le vecteur potentiel de promotion du consensus de Washington dans les administrations financières africaines.

\subsection{Le niveau régional de la coopération intergouvernementale}

C'est le passage d'une coopération intergouvernementale classique à une coopération multiacteurs. La révolution numérique permet de réduire l'écart du point de vue conceptuel entre les pays du Nord, les émergents et les pays du Sud. Mais de par sa capacité à faciliter l'instruction des populations, il engendre aujourd'hui l'impératif de négocier les politiques publiques à plusieurs échelles au sein d'un pays : au niveau de collectivités territoriales, au niveau de la société civile, via le contrôle citoyen de l'action publique ; au niveau de l'Etat central.

Le constat précédent peut être lié à la nature des processus d'innovation numériques en Afrique qui s'apparentent souvent à des processus d'innovation frugale [HAU 16]. Comme par essence ces processus sont contextualisés, il est beaucoup plus difficile à partir d'une capitale de légiférer ou de prendre des décisions, alors même que ces types d'innovation n'existent que par leur dynamique 
d'ancrage territorial local, ce qui traduit une grande diversité de situations techniques et institutionnelles.

Au Burkina, le projet BODI (2015-2019), financé par l'Etat, la Banque Mondiale et l'Open Data Initiative, a permis la création d'une plateforme data.gov.bf. L'objectif est de favoriser la mise à disposition d'informations d'intérêt public. Néanmoins, les chargés de projet font part de leur difficulté à convaincre les différents producteurs de données à les partager. Ce projet également la création d'une plateforme 'Open Election' (http://openelection.data.gov.bf/) qui devrait faciliter l'organisation d'élections libres, transparentes et acceptées par tous.

\subsection{Le niveau du citoyen}

La question du suivi-évaluation, en particulier celle du contrôle citoyen de l'action publique devient de plus en plus incontournable en Afrique subsaharienne. Les technologies numériques permettent aujourd'hui de mettre en évidence le rôle incontournable de l'évaluation, via par exemple les notations des performances des Etats qui sont publiées sur internet par les Institutions internationales comme la Banque Mondiale (CPIA) et même la mise sur des plateformes en ligne des audits affectés dans les différents ministères. Au Togo, a été ainsi mis en ligne en 2017 l'audit effectué par l'Union Européenne, du Ministère Togolais des Finances. Le numérique, favorisant la transparence des informations, devient ainsi pour la société civile un outil de pression sur les Ministères, qui vient s'additionner à la relation classique entre un chef d'Etat ou de Gouvernement et son ministre des finances. Ce n'est plus une instruction de type vertical qui va appeler à une meilleure gouvernance, mais des pressions sociétales sur l'exigence de transparence et de redevabilité. La révolution numérique peut faciliter ce processus de suivi de l'exécution, de l'évaluation et donc de la reddition des comptes. Une des difficultés qui demeure néanmoins est la faible disponibilité des indicateurs de suivi-évaluation, notamment du fait de la faiblesse structurelle des instituts nationaux de statistiques en Afrique.

Sur le plan des mobilisations citoyennes, des organisations de la société civile comme «Yen a marre au Sénégal», le «balais citoyen» au Burkina, ont porté, notamment avec l'appui de la branche ouest-africaine de la Fondation Sorros, l'open society initiative for West Africa (OSIWA), des voix que l'on peut juger incontournables dans la gestion des politiques publiques des pays concernés. Le numérique pourrait donc permettre de renforcer le contrôle citoyen de l'action publique. Mais des auteurs dénoncent les risques de détournement de ce genre de mouvements. Erbs et al (2017) analysant les réseaux d'influence construit en Afrique par le financier Soros, montrent par exemple que les ONG qu'il soutient, dont l'Open Society Initiative, sont des vecteurs de renforcement de l'influence des Etats Unis dans la région et promeuvent des conditions favorables aux investissements du multimilliardaire [ERB 17].

\subsection{La construction d'un projet de société}

Le succès ou l'échec d'une innovation ne peut être dissocié du projet de société au service duquel elle est censée se mettre. Or dans la plupart des pays prédomine de manière croissante ce qu'il est convenu de nommer «le dictat de l'urgence », illustré par la prééminence du court terme au détriment d'une vision concertée sur du moyen/long terme. Or, le travail de construction d'une vision partagée s'avère indispensable car cette dernière constitue au final, le principal filtre de cohérence par lequel on peut évaluer l'opportunité et/ou la qualité du passage à l'échelle d'une innovation, notamment numérique.

\section{Conclusion}

Le développement économique de l'Afrique de l'Ouest est traditionnellement caractérisé par un phénomène de triple extraversion [NUB 07]. L'extraversion est 'réelle' : les ressources, biens et 
services produits localement ne sont pas valorisés pour la consommation interne (avec notamment le cas des cultures de rente ou de l'extraction de métaux, entièrement destinée à l'exportation). L'extraversion est également monétaire et financière : la question de la souveraineté des pays utilisant le FCFA fait débat et les taux d'intérêt prohibitifs offerts par les banques ne sont pas des outils adaptés pour financer l'innovation. L'extraversion est enfin intellectuelle : la portée des innovations frugales, contextualisées, endogènes, qui sont faites dans les territoires est encore sousestimée. La tendance au niveau du pouvoir central demeure de valoriser en priorité ce qui est importé du reste du monde.

Les usages observés du numérique permettent-il de sortir de ce phénomène de triple extraversion ? Ces technologies peuvent-elles participer à une transformation structurelle profonde des économies et sociétés ouest-africaine, en rupture avec les modèles de développement observés jusqu'alors?

Sur la base des expériences empiriques mobilisées (francophonie économique et numérique, Ministère de la prospective et de l'évaluation des politiques publiques, accompagnement des systèmes nationaux d'innovation et de recherche, etc.), nous avons identifié trois domaines où l'usage du numérique pourrait impulser des changements soutenant une transformation structurelle des économies et sociétés. Ces technologies peuvent en effet influencer positivement la création et le partage d'informations et de connaissances ; la création de biens et services innovants (dans une mouvance d'entreprenariat social) ; ou encore la conception des politiques et la gestion de l'action publique, notamment en donnant plus de poids aux citoyens. Cependant, les changements observés à l'heure actuelle restent mineurs. Cela s'explique par un accès encore limité des populations aux TIC de dernière génération, par les problèmes posés par les réseaux d'infrastructures peu développés ou par les difficultés éprouvées pour développer des applications pertinentes au regard des besoins mais également économiquement viables. On observe cependant les prémices d'une transformation systémique identifiable au niveaux de nouveaux réseaux d'acteurs et d'organisations soutenant le déploiement de ces nouveaux usages du numérique.

Il convient alors de questionner la marge de manœuvre que peuvent exercer les porteurs d'innovations frugales et contextualisées face aux grands groupes industriels et firmes globalisées, telles les GAFA (Google, Amazon, Facebook, Apple), aujourd'hui moteur de la transition numérique. Enfin, il convient de rappeler les impacts néfastes de ces technologies sur l'environnement (l'épuisement des métaux rares devenant tout autant problématique que celui des énergies fossiles [GRO 12 ; FLI 13 ; BIH 14]) mais aussi néfastes pour la situation sécuritaire des pays extracteurs, la concurrence sur les ressources structurant de nombreux conflits contemporains.

Le numérique porte le mythe d'une révolution technologique qui serait source de potentielles nouvelles croissances. En réalité, les observations actuelles tendent à infirmer ou du moins à très fortement nuancer cette croyance. La transition numérique entretient potentiellement le mirage historique du développement sociétal par la technique, pourtant déjà dissipé par les travaux en histoire ou sociologie économique [GOD 17]. La révolution numérique pourrait ainsi être un leurre technologique, les TIC n'étant qu'un avatar technologique de plus dans une évolution scientifique technologique en soit continue, mais ne pouvant être la source d'une révolution radicale des modèles de développement. Ces modèles sont structurés par les trajectoires d'industrialisation, de gouvernance macro-politique et/ou de spécialisation qui ont été précédemment identifiées.

Le numérique présente à court et moyen termes des opportunités structurantes d'utopies réalistes pour le développement structurel, s'il répond à des enjeux de biens communs ou d'intérêt public: santé, éducation, entrepreneuriat, gestion des communs, transparence de l'information, participation citoyenne. Mais la transition numérique ne serait porteuse potentielle d'une révolution structurelle pour l'Afrique que dans l'hypothèse où elle serait mise au service des questions que pose le besoin de transformation structurelle des modèles de développement de ce continent, et non de celui des 
firmes globalisées ou des GAFA qui s'en font le moteur et réduisent l'Afrique à une simple opportunité de marché à conquérir.

Cette mise en service du numérique aux besoins du développement ne peut s'opérer que par la construction de cadres de régulation publique (fiscalité, normes, accès aux espaces) des conditions d'accès du continent Africain aux firmes qui portent la transition numérique. La construction de ces cadres implique ensuite les moyens effectifs de leur mise en œuvre et les ressources humaines nécessaires à celles-là.

La capacité à appréhender le degré de convergence ou d'incommensurabilité des relations entre le numérique et le développement est tributaire dans la capacité à prendre en compte les niveaux des politiques nationales, des coopérations inter-gouvernementales, du citoyen et de construction d'un projet de société. Il interpelle comment mieux mobiliser les cadres méthodologiques d'analyses de méthodes systémiques multi niveaux [CHA 19].

On observe par ailleurs que les innovations générées par le numérique dans l'agriculture Africaine sont encore peu nombreuses, souvent exploratoires et restent de faible envergure. La faiblesse structurelle des liens entre d'un côté, la production académique des universités et centres de recherche, et de l'autre, la demande sociétale [TEM 18B] et entrepreneuriale [MIN 18], argumentent pour la multiplication de Think tanks. Ces dispositifs structurent des besoins d'applications concrètes du numérique pour un entrepreneuriat d'innovation inclusif. Ces espaces de réflexion et ces échanges favorisant les actions collectives pourraient permettre de développer des solutions de politiques publiques et d'activer la transformation des résultats de la recherche en technologies sources d'innovations pour un changement de paradigme de développement1. Certes selon Walras « à la limite, ce serait le droit du savant de faire de la science pour la science », mais cela n'est pas incompatible avec le besoin de construire le pont entre la recherche universitaire, la recherche scientifique et les politiques publiques. On constate en Afrique que l'essentiel des politiques d'innovation et de recherche reste structuré par les orientations données par les bailleurs internationaux. Si selon North entre les institutions et les performances, il y a les incitations, que ce soit sur la question de l'innovation ou d'autres éléments clefs du processus d'émergence, la question des incitations devrait être au cœur des réflexions.

Un enjeu supplémentaire tient à la mise en place d'un régime de protection de la propriété intellectuelle, encourageant la créativité et prévenant la 'fuite des talents' qui s'observe actuellement.

Enfin, une des opportunités qu'ouvre la transition numérique, non explorée dans cet article, est de spécifier comment cette transition solidifie l'émergence de marchés intérieurs qui structurent la compétitivité des activités de services mobilisant des ressources localisées dans différents domaines comme l'alimentation, la santé, l'éducation.

\section{Bibliographie}

[AKe 16] AKer, J. C., Ghosh, I., Burrell, J. «The promise (and pitfalls) of ICT for agriculture initiatives", Agricultural Economics, $\mathrm{n}^{\circ} 47$ (S1), p.35-48, 2016.

[AlE 18] ALEXANDRE, C., Technologies de l'information et la communication et accompagnement des agriculteurs en Afrique de l'Ouest: quelles nouvelles configurations des services de conseil agricole? Proposition d'une grille d'analyse. Forum de l'innovation, RRI Nîmes, 04 juin 2018, 04 juin 2018.

[AlE 19] AleXANDRE, C., Emergence du numérique et transformations des services de conseil agricole au Burkina Faso. Conférence AgriNumA, Dakar, 28 au 30 avril 2019.

\footnotetext{
${ }^{1}$ L'auteur de ces lignes a créé en avril 2019 à Lomé (Togo) un Think Tank, « L'Essentiel », dont le but est d'aider à la décision pour la transformation structurelle de l'économie togolaise.
} 
[AMI 73] AMIN, S., Le développement inégal: essai sur les formations sociales du capitalisme périphérique, Editions de Minuit, Paris, 1973.

[Bat 17] Bathelt, H., Cohendet, P., Henn, S., Simon, L., The Elgar Companion to Innovation and Knowledge Creation, Edward Elgar Publishing, Cheltenham, 2017.

[BAY 06] BAYART, J. F., L'Etat en Afrique: la politique du ventre, Fayard, Paris, 2006.

[BIH 14] BIHOUIX, P., L'âge des low tech : vers une civilisation techniquement soutenable, Edition du Seuil, Paris, 2014.

[CAD 16] CADOT, O., DE MElO J., «Introduction. Vers une transformation structurelle en Afrique », Revue d'Economie du développement, vol. 24, $\mathrm{n}^{\circ} 2$, p. 5-17, 2016.

[CAS 15] Casadella, V., Liu, Z., UzUnidis, D. Développement économique et capacités d'innovation dans la mondialisation, ISTE Editions, Londres, 2015.

[CAS 17] CASAdella, V., Temple L., «L'entrepreneur et le développement », dans A. TIRAN et D. UzUNIDIS (eds), Dictionnaire économique de l'entrepreneur, Collection Bibliothèque de l'Economiste, $\mathrm{n}^{\circ} 11$, Éditions Classiques Garnier, Paris, 2017.

[Cha 19] Chanteau, J. P., BorRell T., TeMPLE L., « La conception managériale de la RSE : une innovation sociale ? Enjeux d'une méthode d'évaluation systémique », Revue Innovation, vol. 2, n 59, p. 43-74, 2019.

[DAV 02] DAVIRON, B., GIBBON, P., «Global commodity chains and African export agriculture », Journal of agrarian change, vol. 2, $\mathrm{n}^{\circ} 2$, p. 137-161, 2002.

[DEB 71] DE BERNIS, G., «Les industries industrialisantes et les options algériennes », Revue Tiers Monde, p. 545$563,1971$.

[DUR 17] DURY S., VAll E., IMBERNON J., «Production agricole et sécurité alimentaire en Afrique de l'Ouest ». Cahiers Agricultures, vol. 26, nº, 2017

[EGG 18] EGG, J., GABAS, J.J., GERONIMI, V., « Essai sur l'économie politique du développement: en hommage à Philippe Hugon », Mondes en développement, $\mathrm{n}^{\circ} 4$, p. 137-164, 2018

[Fau 18] Faure, G., Chiffoleau, Y., Goulet F., Temple L., Touzard, J.M., Innovation and development in agricultural and food systems, Éditions Quæ, Versailles, 2018.

[Fli 13] FliPo, F., DoBre, M., Мichot, M., La face cachée du numérique: l'impact environnemental des nouvelles technologies, Éditions L'Échappée Montreuil, 2013.

[Fou 18] FouilleuX E., BRICAS N., AlPha A., «Feeding 9 billion people: global food security debates and the productionist trap », in C. DAUGBJERG Carsten (ed.), P. H. FEINDT (ed.), Transforming food and agricultural policy: Post-exceptionalism in public policy, Oxon, Routledge, 2018.

[FUh 17] FuHrER, C., HoAREAU, É., CUCCHI, A., « Le rôle des Technologies de l'Information et de la Communication dans la dynamique d'un réseau d'innovation: une approche ANT », Revue Innovations, n 3, p. 197-228, 2017.

[GEE 02] GEELS F.W., « Technological transitions as evolutionary reconfiguration processes: a multi-level perspective and a case-study », Research policy, vol. 31, n8, p. 1257-1274, 2002.

[GHE 12] GhernaOuti, S., Aghroum, C., « Cyber-résilience, risques et dépendances: pour une nouvelle approche de la cyber-sécurité », Sécurite et stratégie, vol. 11, n 4, p.74-83, 2012.

[God 17] Godin, B., VINCK, D., Critical studies of innovation: Alternative approaches to the pro-innovation bias, Edward Elgar Publishing, Cheltenham, 2017.

[GRO 12] GROUPE ECOINFO (éd.), Impacts écologiques des technologies de l'information et de la communication : les faces cachées de l'immatérialité, EDP sciences, Paris, 2012.

[HAU 16] HAUDEVILLE, B., LE BAS, C., «L'innovation frugale, paradigme technologique naissant ou nouveau modèle d'innovation? », Revue Innovations, n³, p.9-25, 2016.

[HEE 02] HEEKS, R, « Information Systems and Developing Countries: Failure, Success, and Local Improvisations », The Information Society, vol. 18, n², p.101-112, 2002.

[HIS 13] HisLOP, D., Knowledge Management in Organizations : a critical introduction, Third Edition, Oxford University Press, Oxford, 2013.

[HUG 80] HugON, P., «Dualisme sectoriel ou soumission des formes de production au capital : peut-on dépasser le débat? » Revue Tiers Monde, p. 235-259, 1980.

[LAU 18] LAURENT., E., L'impasse collaborative pour une véritable économie de la coopération, Editions Les liens qui libèrent, Paris, 2018. 
[LOS 12] LOSCH B., Freguin-Gresh S., WhITE E.T., Structural transformation and rural change revisited: challenges for late developing countries in a globalizing world. World Bank, Washington, 2012.

[Mil 19] Mills, J., Reed, M., SkaAlsveen, K., \& IngRam, J., « The use of Twitter for knowledge exchange on sustainable soil management », Soil Use and Management, 2019.

[Min 18] MinKouA NZIE J.R., TEMPLE L., «L'offre de recherche converge-t-elle vers les besoins du secteur agroalimentaire au Cameroun? Une analyse par la bibliographie », Cahier d'Agriculture, 2018.

[Nna 12] Nnadi, F. N., Chikaire, J., Egwuonwu, H. A., Echetama, \& Atoma, C., «ICT for Agriculture Knowledge Management in Nigeria: Lessons and Strategies for Improvement», Science Journal of Agricultural Research and Management, 2012.

[NuB 07] NuBUKPO, K.K., «L'efficacité de la Politique Monétaire en Situation d'Incertitude et d'Extraversion: Le Cas de l'Union Economique et Monétaire Ouest Africaine (UEMOA) ». The European Journal of Development Research, vol. 19, n³, p.480-495, 2007.

[NUB 11] NUBUKPO K., L'improvisation économique en Afrique de l'Ouest : du coton au franc CFA, Editions Karthala, Paris, 2011.

[NUB 12] NUBUKPO K., «Le policy mix de la zone UEMOA : leçons d'hier, réflexions pour demain », Revue Tiers Monde, vol. 4, n²12, p. 137-152, 2012.

[RAZ 17] RAZAFINDRAKOTO, M., ROUBAUD, F., WACHSBERGER, J.M., Institutions, gouvernance et croissance de long terme à Madagascar: l'énigme et le paradoxe, Edition DIAL, Paris, 2017.

[RIC 17] RICHARD, S., «L'apport du secteur privé. Mettre la transformation digitale au service du développement », dans P. CARON et J.M. CHATAIGNER, Un défi pour la planète: Les Objectifs de développement durable en débat, Edition IRD, Marseille, 2017.

[ROG 88] Rogers, E. M., "The Intellectual Foundation and History of the Agricultural Extension Model », Knowledge, vol. 9 n 4, p.492-510, 1988.

[STI 14] STIGLITZ JE, GReENWALD B., La nouvelle société de la connaissance, Éditions Les Liens qui libèrent, Paris, 2014.

[SUC 16] SUCHIRADIPTA, B., SARAVANAN, R., Social media: Shaping the future of agricultural extension and advisory services, GFRAS Working Document, 2016.

[Tem 17] Temple L., Machikou Ndzesop N., Fongang Fouepe G.F., Ndoumbe Nkeng M., Mathe S., « Système national de recherche et d'innovation en Afrique : éclairage du Cameroun ». Revue Innovations, $\mathrm{n}^{\circ}$ 53, p.41-67, 2017.

[Tem 18a] Temple L., Barret D., Blundo Canto G., Dabat MH., DevauX-Spatarakis A., Faure G., Hainzelin E., Mathé S., TOILlier A., Triomphe B., «Assessing Impacts of Agricultural Research for Development: a systemic model focusing on outcomes », Research Evaluation, vol. 27, n², p. 157-170, 2018.

[Tem 18B] TEMPle L., GaunAND A.,Trouche G., Vall E., «Évaluer les impacts des recherches en agriculture sur la société et les écosystèmes : outils, méthodes, études de cas », Cahier d'Agriculture, n²7, 2018

[UZU 11] UZUNIDIS, D., LAPERCHE, B., " The new mercantilism and the crisis of the global knowledge economy », Journal of the Knowledge Economy, vol. 2, n³, 2011.

[VAN 15] VANGala, R. N. K., MUKeRJi, M., \& HiREMATH, B. N., ICTs for agriculture knowledge management: insights from DHRUVA, Proceedings of the Seventh International Conference on Information and Communication Technologies and Development - ICTD n 15, 1-4, 2015. 